\title{
Bragg Grating Fabrication on Tapered Fiber Tips based on Focused Ion Beam Milling
}

\author{
Ricardo M. André*a,b, Martin Becker ${ }^{\mathrm{b}}$, Jan Dellith ${ }^{\mathrm{b}}$, Manfred Rothhardt ${ }^{\mathrm{b}}$, M. I. Zibaii ${ }^{\mathrm{c}}, \mathrm{H}$. Latific, \\ Manuel B. Marques ${ }^{\mathrm{a}}$, Hartmut Bartelt ${ }^{\mathrm{b}}$, Orlando Frazão ${ }^{\mathrm{a}}$ \\ aINESC TEC and Department of Physics and Astronomy, Faculty of Sciences, University of Porto, \\ Rua do Campo Alegre 687, 4150-179 Porto, Portugal \\ ${ }^{b}$ Leibniz Institute of Photonic Technology (IPHT Jena), Albert-Einstein-Straße 9, 07745 Jena, \\ Germany \\ ${ }^{\mathrm{c}}$ Laser and Plasma Research Institute, Shahid Beheshi University, Evin, Tehran, Iran
}

\begin{abstract}
Focused ion beam milling is used on chemically etched tapered fiber tips to create fiber Bragg gratings. These fiber Bragg gratings are based on a modulation of silica and external medium. This leads to a wide and structured spectrum obtained due to imperfections and the inherent structure of the tip. The fiber Bragg gratings presented are very short and have a length of $27 \mu \mathrm{m}$ and $43 \mu \mathrm{m}$ and are milled on the tapered fiber tip. They are characterized in the high temperature range $350-850^{\circ} \mathrm{C}$ and a sensitivity of $14.4 \mathrm{pm} / \mathrm{K}$ is determined.
\end{abstract}

Keywords: Optical fiber sensors, focused ion beam, fiber Bragg gratings, high temperature sensors, tapered fiber tips

\section{INTRODUCTION}

Focused Ion Beam (FIB) is a technology that employs a focused ion beam to image a surface, mill/ablate a structure and even deposit materials [1]. It was initially developed for the semiconductor industry but later became a tool that could be applied in many other fields such as in optical fiber sensors. FIB allows the creation of high resolution structures with nanometric features in any material. Only recently has it been employed to create optical fiber sensors such as Fabry-Perot cavities for temperature [2] and refractive index sensing [3], fiber top cantilevers for atomic force microscopy [4], and microwire cantilevers for vibration sensing [5]. Although possible, milling large quantities of material takes a very long time. To overcome this shortcoming, it is necessary to pre-process the fiber with a different technique in order to have a smaller structure and an easier access to the light guiding region of the fiber. Several techniques have been used to facilitate the FIB milling process: $\mathrm{CO}_{2}$ laser tapering [6], chemical etching of special structure forming fibers [5], polishing [7], and others. Fiber Bragg gratings (FBGs) have also been created with FIB by Kou et al: : both an FBG on a tapered fiber tip for temperature sensing [6] and a metal-dielectric-hybrid fiber tip grating for refractive index sensing [8]. These solutions don't explore the high temperature range that these structures allow.

In this work, the chemical etching of a standard single mode fiber is employed to create tapered fiber tips and consequently, quickly reduce the size of the structure, making the FIB milling of gratings possible. On these tapered fiber tips, different length fiber Bragg gratings are milled using the focused ion beam. A spectral comparison of a 50-period-long grating when immersed in air and in ethanol is performed. A second, 80-period-long grating, is characterized as a high temperature sensor in the range $350-850^{\circ} \mathrm{C}$.

*randre@inescporto.pt;

24th International Conference on Optical Fibre Sensors, edited by Hypolito José Kalinowski,

José Luís Fabris, Wojtek J. Bock, Proc. of SPIE Vol. 9634, 96343I · (c) 2015 SPIE

CCC code: $0277-786 \mathrm{X} / 15 / \$ 18 \cdot$ doi: $10.1117 / 12.2194978$

Proc. of SPIE Vol. $9634963431-1$ 


\section{EXPERIMENTAL RESULTS}

Focused Ion Beam (FIB) is a technology that relies on an ion beam to image or ablate a surface very much like a scanning electron microscope, where instead of an electron beam an ion beam is used. The ions have a much larger mass and therefore mill the surface on which they are focused. Controlling the ion current and the spot size, it is possible to create very small structures with a very high resolution. One limitation of this technique is that in order to achieve high resolution and mill nanometric structures, a low current is need. This makes it practically impossible to mill FBGs in standard 125 $\mu \mathrm{m}$ fiber, as to reach the light guiding section, one would have to mill $60 \mu \mathrm{m}$-deep slices with just a few hundred nanometers of width, which would amount to an impossible to achieve aspect ratio. Therefore, a pre-processing of the fiber is necessary to create an intermediate structure on which it is possible to mill gratings with FIB. In this work, tapered fiber tips were chosen due to the ease of fabrication and their proven applicability. Dynamic HF chemical etching was used to fabricate the TFTs by controlling the oil-acid interface relative to the fiber. This level is controlled by changing the volume of the acid using a syringe pump, achieving tips with selectable angles and smooth surfaces [9].
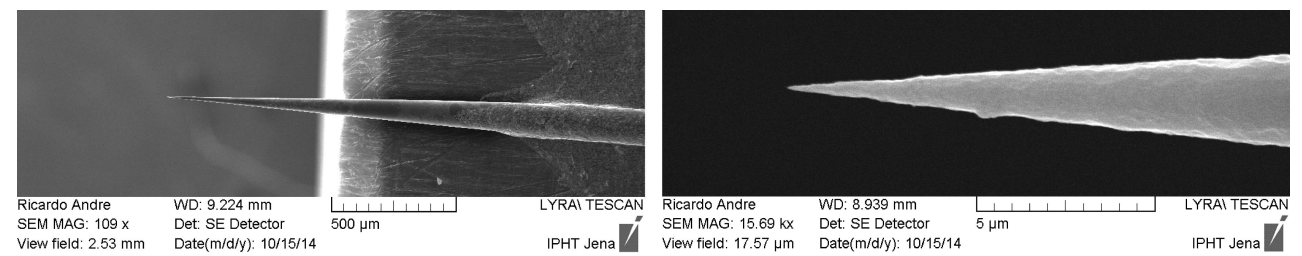

Figure 1. SEM micrographs of tapered fiber tips

\section{Fiber Bragg Grating Fabrication with Focused Ion Beam}

Milling fiber Bragg gratings with FIB is not trivial. Many problems arise when trying to create such small and detailed structures in non-conducting materials such as glass fibers. In an attempt to avoid charging problems such as beam deviation and sample charging and consequent vibration, a thin tantalum film (ca. $50 \mathrm{~nm}$ ) is deposited on the TFTs. This will allow the sample to superficially conduct and prevent it from negatively charging from the electron beam and positively charging from the ion beam. In Figure 2 one can see an example of a TFT-FBG milled with FIB. This FBG has 50 periods and a total length of just $27 \mu \mathrm{m}$. Each period is $540 \mathrm{~nm}$ and each indentation has only $270 \mathrm{~nm}$. Very low ion beam currents and consequently small spot sizes are necessary to mill with such high precision as to maintain the structural integrity of each indentation. The milling of a 50-period grating takes an average time of 50 min using a current of 115 pA. All structures were milled using the LYRA FIB-SEM system from TESCAN. Longer gratings can be achieved but other problems need to be overcome. One problem is that with a longer processing time, a drift of the beam/sample occurs leading to gratings such as the one shown in Figure 3. These gratings, even having a drift, still function in the same way as expected. This drift can however, be preemptively corrected using a gradual counter-drift in the initial designing of the grating.
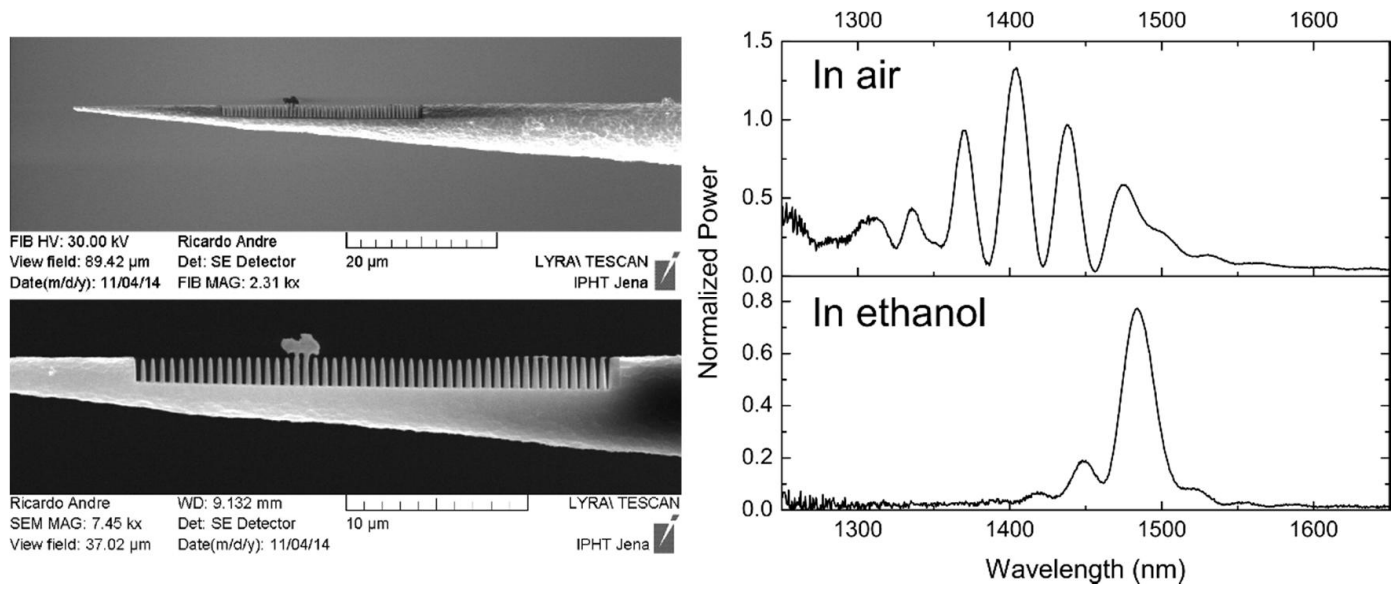

Figure 2. Micrographs of a FIB-milled 50-period TFT-FBG (left) and corresponding spectra in air (top right) and ethanol (bottom left). 
These gratings, due to the inherent geometry of the fiber tip, must be analyzed in reflection using a simple setup comprised of a light source, an optical circulator and an optical spectrum analyzer. The source used was a supercontinuum light source due to the broad nature of the Bragg peaks and to fully grasp the full spectrum of the light reflected by the Bragg grating. The grating in Figure 2 has been analyzed in reflection when in air and when dipped in ethanol. One can easily observe a red-shift and an abrupt change in the spectral shape. The red-shift can be accounted for by the change in effective refractive index of propagation when dipped in the different media. The refractive index of air is 1.00 as compared to ethanol which is 1.35. This leads to a higher Bragg wavelength when the grating is dipped in the higher refractive index medium. The fringed spectrum of the grating in air is likely due to imperfections in the grating that cause phase shifts and several reflections along the grating. When the grating is dipped in ethanol, the index contrast is much lower $(1.35 / 1.45 \mathrm{instead}$ of $1.00 / 1.45)$ and these imperfections and unwanted reflections are minimized resulting in a narrower peak.

In Figure 3, a micrograph of a FIB-milled 80-period grating ( $43 \mu \mathrm{m}$ in length) is shown where a drift towards the central section of the TFT occurred during fabrication. This drift did not present significant changes to the FBG spectrum as shown in Figure 4. This grating was subjected to temperature variations in the range $350^{\circ} \mathrm{C}-850^{\circ} \mathrm{C}$ as can also be seen in Figure 4. The reflectivity of the grating is a bit reduced at high temperatures but restores itself when the temperature decreases. A sensitivity of $14.4 \mathrm{pm} / \mathrm{K}$ was achieved.

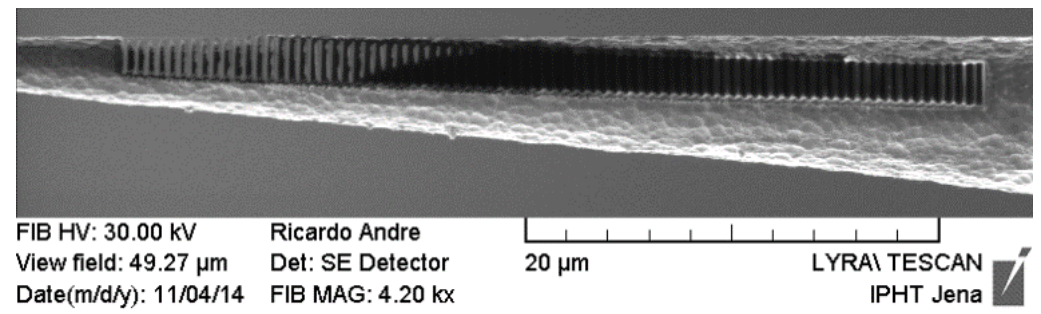

Figure 3. Micrograph of a FIB-milled 80-period TFT-FBG where a drift to the center of the fiber occurred during production.
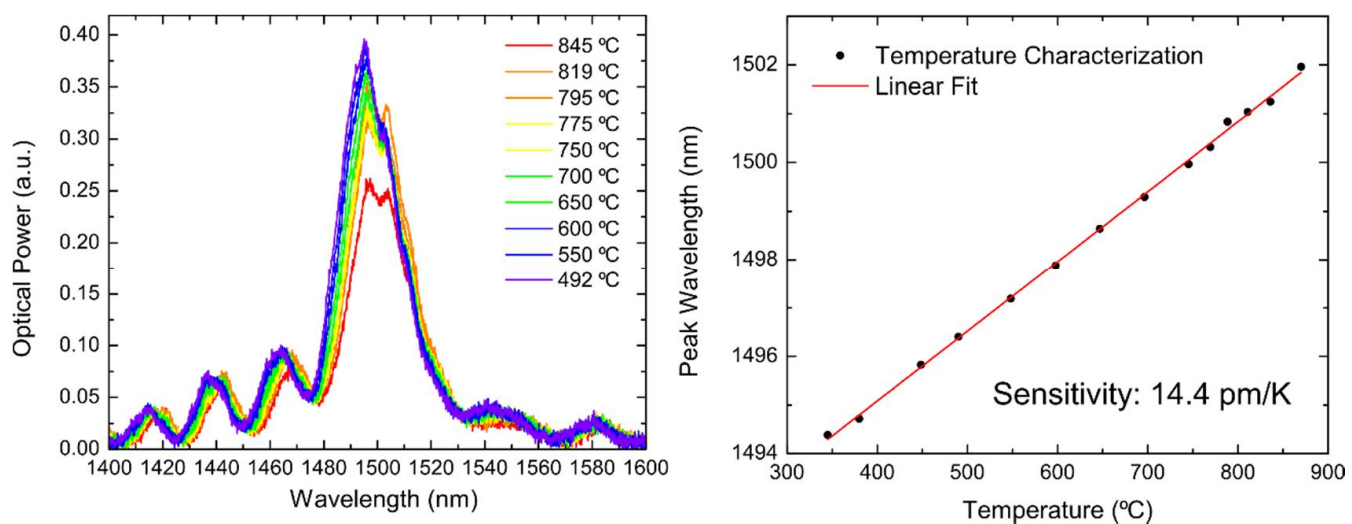

Figure 4. High temperature characterization of the 80-period TFT-FBG and temperature response with a sensitivity of $14.4 \mathrm{pm} / \mathrm{K}$. 


\section{CONCLUSIONS}

Different fiber Bragg gratings were created on chemically etched tapered fiber tips. The 50-period-long TFT-FBG was shown to have very different spectra when in air and ethanol. The lower refractive index of ethanol when compared to air led to a lower effective index of the grating and a reflection at a higher wavelength. A cleaning of the fringes and narrowing of the peak also resulted from the lower index contrast when the grating was immersed in ethanol. The 80-period-long TFT-FBG, was characterized as a high temperature sensor in the range $350-850^{\circ} \mathrm{C}$ and showed a higher sensitivity of 14.4 $\mathrm{pm} / \mathrm{K}$ when compared to standard FBGs.

The high temperature performance of such a simple structure comes from the fact that the index modulation is geometric in nature. It does not depend on a slight index change, result of more traditional fabrication techniques such as the use of excimer lasers or femtosecond lasers that can be erased at high temperatures. This means that the grating will only be erased when the silica starts to soften and the periodic indentations merge together. The modulation in this type of grating is made of consecutive slices of silica and external medium (such as air) making possible a refractive index contrast of over $10^{-1}$. This also allows for much shorter gratings which combined with the tip-like structure of the tapered fiber tips leads to a very interesting high temperature point sensor. Many applications can benefit from such structures. Temperature /refractive index measurement in very small volumes such as bubbles or even cells can be two examples. Other gratings or Fabry-Perot cavities can be combined on these TFTs for multiplexing and probing different parameters and wavelengths. All this without compromising the size of the sensor tip.

\section{ACKNOWLEDGMENTS}

The work of Ricardo André was supported in part by Fundação para a Ciência e Tecnologia under the grant SFRH/BD/84048/2012. Project "NORTE-07-0124-FEDER-000058" is financed by the North Portugal Regional Operational Programme (ON.2 - O Novo Norte), under the National Strategic Reference Framework (NSRF), through the European Regional Development Fund (ERDF), and by national funds, through the Portuguese funding agency, Fundação para a Ciência e a Tecnologia (FCT).

\section{REFERENCES}

[1] Reyntjens, S.., Puers, R., “A review of focused ion beam applications in microsystem technology,” J. Micromechanics Microengineering 11(4), 287-300 (2001).

[2] Kou, J., Feng, J., Ye, L., Xu, F.., Lu, Y., "Miniaturized fiber taper reflective interferometer for high temperature measurement," Opt. Express 18(13), 14245-14250, (2010).

[3] Kou, J., Feng, J., Wang, Q., Xu, F.., Lu, Y., "Microfiber-probe-based ultrasmall interferometric sensor," Opt. Lett. 35(13), 2308, (2010).

[4] Iannuzzi, D., Deladi, S., Schreuders, H., Slaman, M., Rector, J. H.., Elwenspoek, M., "Fiber-Top Cantilevers: A New Generation of Micromachined Sensors for Multipurpose Applications - OSA Technical Digest (CD)," Opt. Fiber Sensors, TuB2, (2006).

[5] André, R. M., Pevec, S., Becker, M., Dellith, J., Rothhardt, M., Marques, M. B., Donlagic, D., Bartelt, H.., Frazão, O., "Focused ion beam post-processing of optical fiber Fabry-Perot cavities for sensing applications.," Opt. Express 22(11), 13102-13108, (2014).

[6] Kou, J., Qiu, S., Xu, F.., Lu, Y., "Demonstration of a compact temperature sensor based on first-order Bragg grating in a tapered fiber probe,” Opt. Express 19(19), 18452, (2011).

[7] Wieduwilt, T., Dellith, J., Talkenberg, F., Bartelt, H.., Schmidt, M. A., "Reflectivity enhanced refractive index sensor based on a fiber-integrated Fabry-Perot microresonator.," Opt. Express 22(21), 25333-25346, (2014).

[8] Kou, J.-L., Qiu, S.-J., Xu, F., Lu, Y.-Q., Yuan, Y.., Zhao, G., "Miniaturized Metal-Dielectric-Hybrid Fiber Tip Grating for Refractive Index Sensing," IEEE Photonics Technol. Lett. 23(22), 1712-1714 (2011).

[9] Nikbakht, H., Latifi, H., Amini, T.., Chenari, Z., "Controlling cone angle of the tapered tip fiber using dynamic etching," OFS2014 23rd Int. Conf. Opt. Fiber Sensors, J. M. López-Higuera, J. D. C. Jones, M. López-Amo, and J. L. Santos, Eds., 91574Q, International Society for Optics and Photonics (2014). 